\title{
Characterization of Nonmotile Neutrophil Subpopulations in Neonates and Adults
}

\author{
PETER J. KRAUSE, DONALD L. KREUTZER, LEONARD EISENFELD, VICTOR C. HERSON, \\ STEVEN WEISMAN, PHYLLIS BANNON, AND NADIA GRECA \\ The Departments of Pediatrics. The Pediatric Research Laboratory, IIartford IIospital and The L'niversity of \\ Connecticut School of Medicine, and The Departments of Pediatrics and Pathology, The University of \\ Connecticut School of Medicine, Farmington, Connecticut 06115
}

\begin{abstract}
Previous studies have demonstrated that polymorphonuclear leukocytes (PMN) are not a homogeneous population of cells but differ significantly in their structure and function. PMN move at varying rates, and a fraction estimated from 20 to $70 \%$ do not move at all in response to chemotactic stimuli. To characterize this PMN subpopulation better, we studied PMN motility in neonates and adults using a polycarbonate micropore filter chemotactic assay and the 31D8 MAb. Most PMN strongly bind 31D8 MAb (31D8 "bright"), but a minority (31D8 "dull") weakly bind the antibody and in this respect are similar to immature PMN precursors. The 31D8 "dull" PMN have impaired function compared with 3108 "bright" PMN. In the present study, a PMN subpopulation that failed to migrate using the micropore filter assay accounted for $\mathbf{5 8}$ $\pm 7 \%$ of adult $\mathbf{P M N}$ and was similar to the migrating subpopulation in regard to viability and phagocytic function. The nonmotile subpopulation had a higher percentage of bands $(5 \pm 3 \%$ versus $1 \pm 2 \%, p<0.01)$ and decreased binding of $31 \mathrm{D} 8 \mathrm{MAb}$ compared with the motile subpopulation. Neonates had a larger nonmigrating PMN subpopulation and 3108 "dull" PMN subpopulation than those of adults $(76 \pm 3 \%$ versus $58 \pm 7 \%, p=0.04$ and $26 \pm$ $11 \%$ versus $8 \pm 2 \%, p<0.01$, respectively). These data indicate that although PMN appear morphologically as a homogeneous population of cells, there exists a viable, nonmotile PMN subpopulation that may be less mature than the motile PMN subpopulation. They also indicate that impaired neonatal PMN motility may be attributable in part to an increased size of the nonmotile PMN subpopulation. (Pediatr Res 25:519-524, 1989)
\end{abstract}

\section{Abbreviations}

PMN, polymorphonuclear leukocytes

NBT, nitroblue tetrazolium

fMLP, f-methionylleucylphenylalanine

$31 \mathrm{D} 8 \mathrm{MAb}$, mouse IgG1 31D8 MAb

PMN generally have been assumed to be a homogeneous cell population despite studies as early as the 1920's showing that there were differences among PMN in their ability to move and to ingest bacteria $(1-2)$. In the past 10 years, numerous studies

Received August 15, 1988: accepted December 9, 1988

Correspondence and reprint requests Peter J. Krause, M.D. Department of Pediatrics. Hartford Hospital, 80 Seymour Street. Hartford. CT 06115.

Supported in part by Hartford Hospital Free Funds and National Institutes of Health Grant EY04131. of PMN structure and function have confirmed that PMN are a heterogeneous group of cells (3-10). It has previously been shown that PMN from adults differ in their ability to move and that a percentage do not move at all in response to chemotactic stimuli (1, 3-6). Estimates of the size of the poorly motile PMN subpopulation have varied, and the characteristics of this PMN subpopulation have not been well described. To further characterize PMN chemotactic subpopulations, we studied PMN motility in healthy adults and in newborn infants using a polycarbonate filter chemotactic assay and two cell surface MAb (31D8 and Mo-1) binding assays. The 31D8 MAb binds PMN heterogeneously and is associated with PMN motility $(9-10)$. Mo-1 binds to a cell surface glycoprotein which helps mediate PMN adherence (11-13). A poorly motile PMN subpopulation was identified in adults and neonates and was found to be larger in neonates. Poorly motile PMN were noted to be viable cells that have similar phagocytic function but decreased binding of 31D8 and Mo-1 MAb compared with motile PMN.

\section{MATERIALS AND METHODS}

Study population. Blood was obtained from the fetal side of placentas (neonatal cord blood) within $5 \mathrm{~min}$ of birth. Placentas were obtained from healthy women who had delivered vaginally or by repeat cesarian section who had local anesthesia, no complications of pregnancy, labor, and delivery and whose newborns were in good health. Blood was also obtained from adult volunteers on no medication. All blood samples were immediately mixed with preservative-free sodium heparin $(50 \mu / \mathrm{mL}$ blood $)$ and brought to the laboratory for analysis. Informed consent to sample blood was obtained from all parents or adult subjects in accordance with Hartford Hospital Institutional Review guidelines.

PMN locomotory assay. Whole blood was purified using $6 \%$ Hetastarch (McGaw Laboratories, Irvine, CA) that yielded a population of cells composed primarily of PMN and monocytes as previously described (14). In some cases, a more purified PMN population was isolated using Hetastarch followed by FicollHypaque separation. PMN locomotory response was measured using a modified polycarbonate micropore filter assay (10). In brief, $5 \times 10^{5} \mathrm{PMN} / 0.7 \mathrm{~mL}$ were placed in the upper compartment of a modified Boyden chamber (Ahlco Corporation, Southington, $\mathrm{CT}$ ) over a $10-\mu \mathrm{m}$ thick polycarbonate filter (Nucleopore, Pleasanton, CA) with a pore size of $5 \mu \mathrm{m}$. Stabilized zymosanactivated normal serum $(3 \%)$ was placed in the lower compartment. The PMN were allowed to penetrate the filter during a 15 min to 7-h incubation in a humid $5 \% \mathrm{CO}_{2}$ atmosphere at $37^{\circ} \mathrm{C}$. PMN were then separately removed from the upper and lower compartments and counted. In some cases, further analysis of these cells was carried out. To determine whether the PMN in the upper compartment were inhibited from entering the lower 
compartment because of plugging of the polycarbonate filter, the assay was repeated using "plugged" filters. "Plugged" micropore filters were prepared by allowing adult PMN to traverse polycarbonate filters for $120 \mathrm{~min}$. The upper and lower compartments were then evacuated, and the micropore filter was gently transferred to a new chemotactic chamber. A second PMN aliquot of $500000 \mathrm{PMN} / \mathrm{mL}$ was then placed in the top compartments over these "used" micropore filters. The same concentration of PMN was simultaneously placed in other chambers containing fresh micropore filters. The number of PMN in the upper and lower chambers was then assessed under both conditions after a 60-min incubation. To further assess whether there was plugging of the micropore filter, adult and neonatal PMN were removed from the top compartment of chemotactic chambers and transferred into new chambers, and the assay was repeated. To make certain there was no movement of PMN from the bottom into the top chamber, PMN were placed in the bottom compartment with or without chemoattractant, the upper chamber evacuated after 45 and $180 \mathrm{~min}$, and a cell count performed.

Trypan blue assay. PMN were tested for viability using a standard trypan blue exclusion assay (15). In brief, $10 \mu \mathrm{L}$ of PMN and $10 \mu \mathrm{L}$ of trypan blue solution $(0.1 \%$ trypan blue in $0.85 \%$ saline) were placed on a slide and a coverslip was added. The slide was placed in a humidified $37^{\circ} \mathrm{C}, 5 \% \mathrm{CO}_{2}$ incubator for $10 \mathrm{~min}$. The slide was then placed under a microscope (magnification, $400 \times$ ), and 100 cells were counted for viability. PMN without a blue-staining nucleus were considered viable; PMN with blue-staining nuclei were counted as dead cells.

NBT assay. PMN were incubated in a mixture of NBT and phorbol myristate acetate for $25 \mathrm{~min}$ at $37^{\circ} \mathrm{C}$. Glass slides were spotted with $20 \mu \mathrm{l}$ of these mixtures in a $37^{\circ} \mathrm{C}$ incubator for 15 min. The slides were removed; a coverslip was placed on top; and 100 cells were counted for the percentage of PMN that reduced NBT (15).

Adherence assay. PMN adherence was determined using a previously described glass coverslip assay (16). In brief, $20 \mu \mathrm{l}$ of PMN $\left(5 \times 10^{6} / \mathrm{mL}\right)$ were placed on a clean, $22-\times 22-\mathrm{mm}$ glass cover slip (Rochester Scientific Co., Inc., Rochester, NY) and incubated $45 \mathrm{~min}$ at $37^{\circ} \mathrm{C}$ in a humid chamber. After incubation, the coverslip was gently rinsed, leaving a thin layer of PMN adherent to the glass. The coverslip was stained with Wright's stain and mounted on a glass slide. PMN were counted in every other field $(0.25-\times 0.25-\mathrm{mm}$ grid; magnification $400 \times)$ along a horizontal and vertical diameter of the circle of adherent PMN. The percentage of PMN adherence was then calculated as follows:

$\% \mathrm{PMN}$ adherence $=(\mathrm{PMN}$ counted $) /($ area counted $) \times$ area of entire circle/PMN in original $20-\mu \mathrm{L}$ sample $\times 100$.

Preparation and MAb labeling of PMN. PMN obtained from the upper and lower compartments of the chemotactic chambers as well as control PMN (not placed in the chemotactic chambers) were analyzed for $31 \mathrm{D} 8$ or Mo-1 MAb binding as previously described $(10,13)$. The $31 \mathrm{D} 8 \mathrm{MAb}$ was a generous gift of $\mathrm{Dr} \mathrm{H}$. Malech, National Institutes of Health, Bethesda, MD (9). The Mo-1 MAb was obtained commercially (Coulter Electronics Corp., Hialeah, FL). In brief, each leukocyte-rich fraction was placed in PBS-BSA, counted, mixed with MAb, and allowed to incubate on ice for $45 \mathrm{~min}$. The PMN were then washed three times in PBS-BSA and incubated for $30 \mathrm{~min}$ on ice with fluorescein-isothiocyanate-labeled goat-antimouse IgG. The labeled PMN were washed three more times in PBS-BSA and stored on ice, in the dark, and in the absence of $\mathrm{Ca}^{2+}$ or $\mathrm{Mg}^{2+}$ until use. Separated and labeled cells remained alive as shown by trypan blue exclusion.

Analysis by flow cytometry. Flow microfluorometry was performed using an EPICS C (Coulter Electronics, Inc., Hialeah, FL) with an argon laser emitting $500 \mathrm{~mW}$ at $488 \mathrm{~nm}$. Forward and $90^{\circ}$ light scatter were used to identify the PMN population from lymphocytes and monocytes. The fluorescence intensity distribution of $10000 \mathrm{PMN}$ was determined. The data were presented as the number of cells on the vertical axis and log green fluorescence on the horizontal axis divided into a three log scale consisting of 255 channels.

Statistical analysis. Statistical significance of differences between groups was performed using the Student's two Tailed $t$ test. A $p$ value of $<0.05$ was considered significance in all cases.

\section{RESULTS}

$P M N$ heterogeneity detected with the polycarbonate micropore filter chemotactic assay. Adult and neonatal PMN were placed in the upper compartments of chemotactic chambers and allowed to move through polycarbonate filters into the lower compartments during incubation times of 15 to $240 \mathrm{~min}$. The results of these experiments are shown in Figure 1. A PMN subpopulation that was recovered in the upper compartment and thus failed to migrate into the lower compartment was noted both in adults and neonates. After $90 \mathrm{~min}$ of incubation, the percentage of $\mathrm{PMN}$ in adults and neonates that failed to migrate did not change significantly even when the incubation times were increased to 420 min (data not shown). The percentage of nonmigrating PMN was larger in neonates than in adults at the following incubation times: $45 \min (90 \pm 3 \%$ versus $67 \pm 3 \%, p<0.01), 90 \min (80$ $\pm 4 \%$ versus $73 \pm 5 \% p=0.14), 180 \min (79 \pm 3 \%$ versus $60 \pm$ $6 \%, p<0.02)$ and $240 \min (76 \pm 3 \%$ versus $68 \pm 7 \%, p<0.04)$.

To examine alternative explanations for recovery of PMN in the upper compartment other than an intrinsic cellular defect, several further experiments were carried out (see "Materials and Methods"). We first determined whether plugging of the micro-
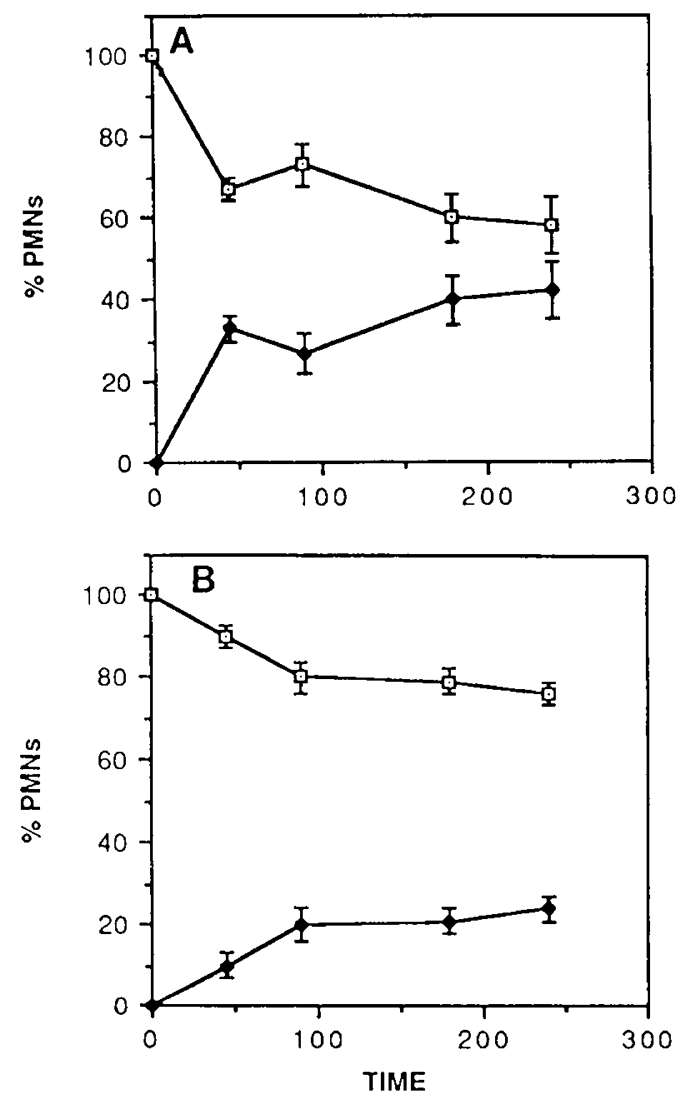

Fig. 1. PMN chemotactic heterogencity in neonates and adults using polycarbonate filters. The mean percentage $( \pm 1$ SEM) of PMN from seven adults $(A)$ and seven neonates $(B)$ recovered from the upper compartments $(\square$, nonmigrating $\mathrm{PMN})$ and lower compartments $(\bullet$, migrating PMN) of chemotactic chambers after incubation times of $15-$ 240 min are shown. After 90 min of incubation, the percentage of PMN in neonates and adults that failed to migrate did not change significantly. 
pore filter ("used" filter) might have prevented PMN movement into the lower compartment. There was no significant difference in the number of PMN recovered from the upper compartments of chemotactic chambers containing "used" and fresh micropore filters. Furthermore, less than $10 \%$ of PMN recovered from the upper compartment after 60 -min incubations and placed in new chemotactic chambers were recovered from the lower compartment after 45- and 180-min incubations. These experiments indicated there had not been plugging of the polycarbonate micropore filter. No PMN were recovered from the upper compartment after placement in the lower compartment and incubating for 45 and $180 \mathrm{~min}$ with or without chemoattractant, indicating PMN did not move from the lower into the upper compartment. Finally, to determine whether motility was chemoattractant-dependent, the polycarbonate micropore chemotactic assay was repeated using $10^{7}-\mathrm{M}$ fMLP in addition to activated serum (C5a) in the lower compartment. The fMLP was added separately and together during a 180 -min incubation in different experiments. There was a larger nonmigrating PMN subpopulation using fMLP $(80 \pm 17 \%)$ than C5a $(54 \pm 18 \%, p$ $<0.014)$. The percentage of nonmigrating PMN was least when C5a and $\mathrm{AMLP}$ were used together $(48 \pm 21 \%)$, although the difference compared with $\mathrm{C5a}$ alone was not significant ( $p=$ $0.18)$.

Characterization of the migrating and nonmigrating PMN subpopulations. To characterize the migrating and nonmigrating PMN subpopulations, adult PMN were placed in the upper compartment of chemotactic chambers, allowed to traverse polycarbonate filters in response to activated normal serum over 45- to 180-min incubation periods, and harvested from the upper (nonmigrating PMN) and lower (migrating PMN) compartments. PMN placed in chemotactic chambers with chemoattractant but without micropore filters were used as controls. The cells obtained were examined for viability with the trypan blue assay, adherence with the glass coverslip adherence assay, phagocytosis with the NBT assay, cell age with the band count, and cell surface antigen with the 31D8 and Mo-1 assays.

The ability of PMN to exclude the dye trypan blue from their nuclei has been used to assess PMN viability. The mean percentage of PMN that excluded trypan blue after $45-\min (n=8)$ and 180-min $(n=8)$ incubations were as follows: nonmigrating PMN, $98 \pm 1 \%$ and $98 \pm 2 \%$, respectively, and migrating PMN, $97 \pm 3 \%$ and $98 \pm 2 \%$, respectively. Thus, both nonmigrating and migrating PMN were viable cells as assessed by trypan blue exclusion.

No significant difference was noted in PMN adherence after a 45 -min incubation $(n=5)$ between the migrating PMN $(20 \pm$ $8 \%$ ) and nonmigrating PMN ( $32 \pm 8 \%$ ), nor was there any difference in adherence between these PMN after $180 \mathrm{~min}$ of incubation ( $n=5,22 \pm 14 \%$ and $17 \pm 4 \%$, respectively).

The NBT assay is a standard screening test for the capacity of PMN to undergo oxidative metabolism. There was no significant difference in NBT reduction between migrating and nonmigrating PMN after 45- and 180-min incubation periods. The mean percentage of PMN that reduced NBT at $45 \mathrm{~min}(n=7)$ and $180 \min (n=7)$ were nonmigrating PMN $99 \pm 2 \%$ and $94 \pm$ $5 \%$, respectively, and migrating PMN $100 \pm 1 \%$ and $99 \pm 1 \%$, respectively.

A larger percentage of bands was noted in the nonmigrating than in the migrating adult PMN subpopulation after $45 \mathrm{~min}$ of incubation $(9 \pm 7 \%$ and $0.3 \pm 0.5 \%$, respectively, $p<0.01, n=$ $13)$ and $180 \mathrm{~min}$ of incubation $(5 \pm 3 \%$ and $1 \pm 2 \%, p<0.01$, respectively, $n=12)$. Similar data were obtained in neonatal PMN for $45-\min (12 \pm 4 \%$ and $1 \pm 3 \%$, respectively, $n=13, p$ $<0.01)$ and 180 -min incubations $(10 \pm 3 \%$ and $1 \pm 2 \%$, respectively, $n=3, p<0.05$ ).

Previous studies have shown that the 31D8 MAb binds to human PMN in a heterogeneous manner and the degree of PMN binding correlates with PMN function. The majority of PMN avidly bind (31D8 "bright"), depolarize and respond chemotac- tically to formyl peptide while a minority weakly bind (31D8 "dull") and fail to respond similarly. PMN from seven adults and seven neonates were tested for 31D8 MAb binding. Neonates had a larger percentage of 3108 "dull" cells $(26 \pm 11 \%)$ than adults $(8 \pm 2 \%, p<0.01)$. Representative $31 \mathrm{D} 8 \mathrm{MAb}$ binding curves are shown in Figure 2.

To characterize migrating and nonmigrating PMN further, cells from the upper (nonmigrating) and lower (migrating) chemotactic chambers were harvested and labeled with 31D8 MAb after 45-, 60-, 180-, and 240-min incubations. The results are summarized in Figure 3 (representative 31D8 MAb binding curves) and in Table 1, where data from 45- and 180-min incubation times are summarized. In both adults and neonates, there was significantly decreased 31D8 MAb binding as determined by mean fluorescence channel in nonmigrating PMN compared with migrating PMN. Control PMN were intermediate in regard to $31 \mathrm{D} 8 \mathrm{MAb}$ binding between the other two groups. The differences in 31D8 MAb binding between nonmigrating and migrating PMN were also apparent when the percentage of 31D8 "dull" cells were calculated. A larger percentage of 31D8 "dull" PMN were noted in the nonmigrating PMN compared with the migrating PMN in both neonates and adults although only the difference in neonatal PMN was statistically significant (Table 1).

The Mo-1 antigen is one of a related group of cell surface glycoproteins that includes the iC 3 b receptor (CR3), LFA-1, and p150,95. Adherence of PMN to cell surfaces is mediated in part
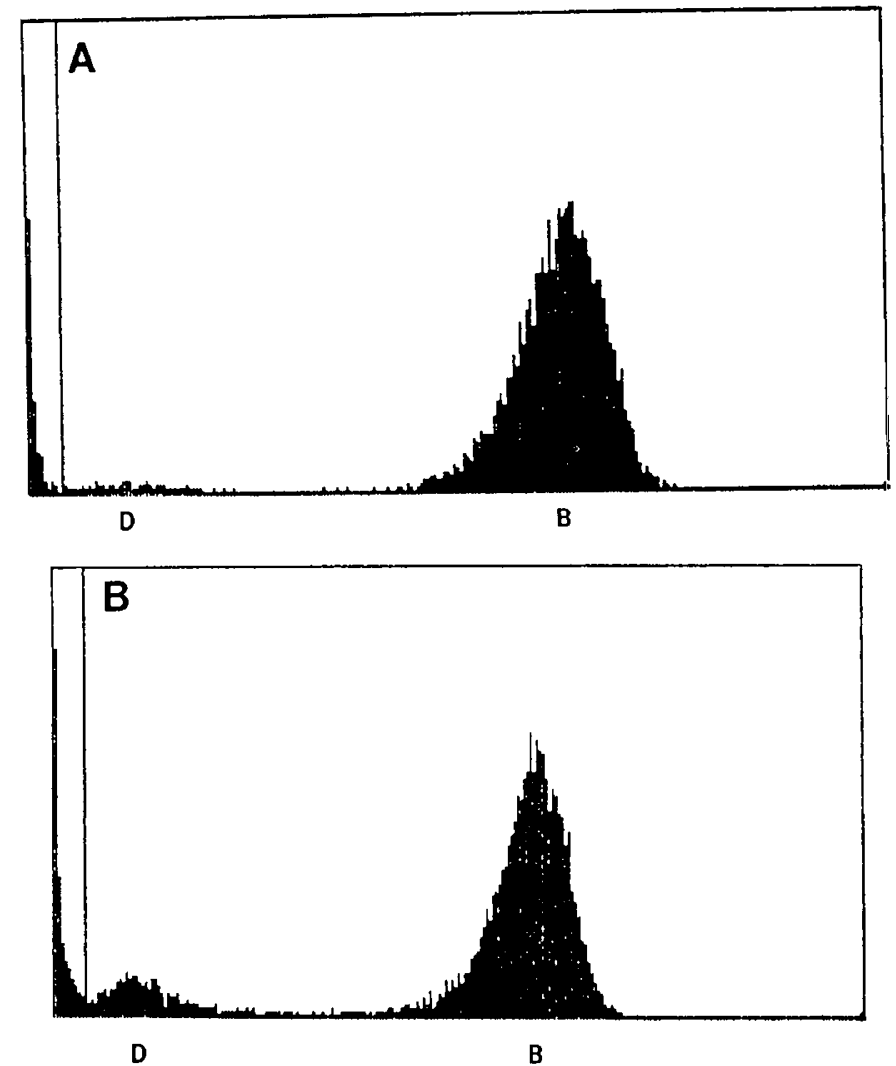

Fig. 2. $31 \mathrm{D} 8$ antibody binds $\mathrm{PMN}$ heterogeneously. The illustrations are representative curves of $31 \mathrm{D} 8 \mathrm{MAb}$ binding to PMN in adults $(A)$ and neonates $(B) . A$ shows a major peak consisting of $31 \mathrm{D} 8$ "bright" PMN ("B") and a minor peak consisting of 31D8 "dull" PMN ("D"). $B$ shows the 31D8 "bright" peak is smaller and the 31D8 "dull" peak is larger. The horizontal axis depicts fluorescence intensity with cells to the left of the vertical line exhibiting autofluorescence, the " $D$ " area one log fluorescence and the " $\mathrm{B}$ " area two log fluorescence. The vertical axis depicts the number of PMN. The percentage of dull cells are $5 \%$ in $A$ and $15 \%$ in $B$. 


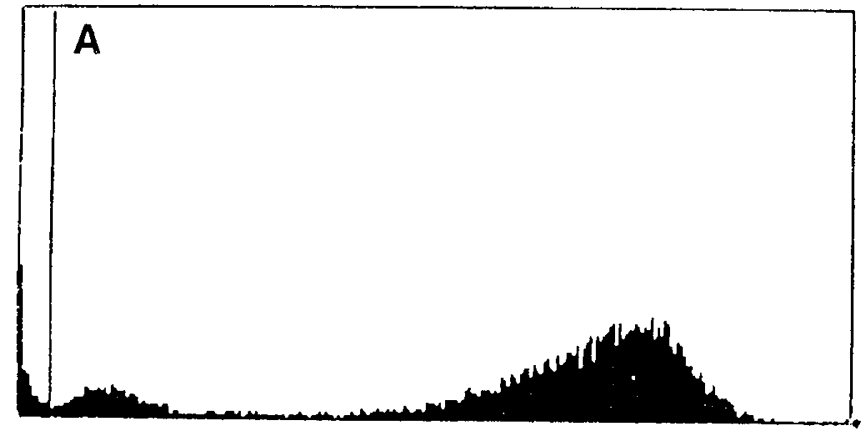

$\square$

B

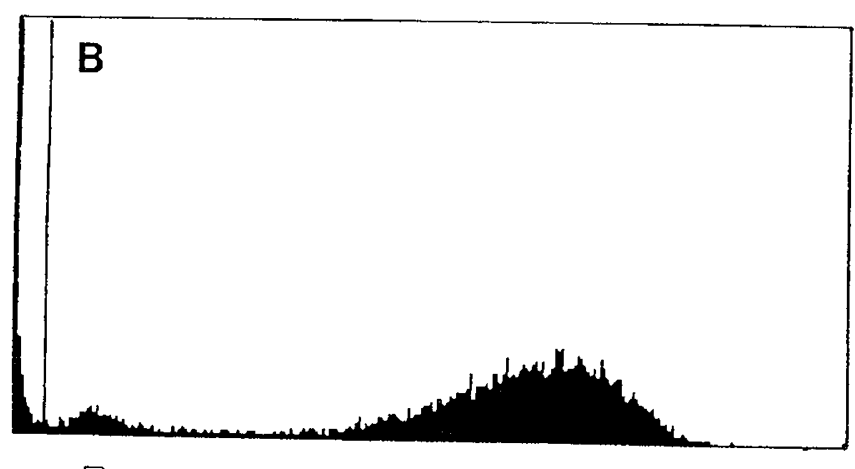

B

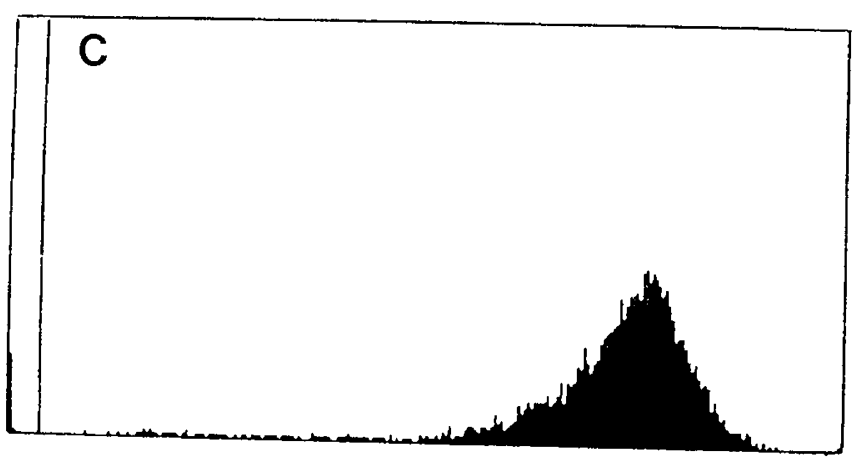

B

Fig. 3. A nonmotile PMN subpopulation has a decreased $31 \mathrm{D} 8 \mathrm{MAb}$ binding compared with the motile PMN subpopulation or total PMN population. In a representative experiment, neonatal PMN aliquots were labeled with $31 \mathrm{D} 8 \mathrm{MAb}$ directly (Control, $A$ ) and after $180 \mathrm{~min}$ incubation in a Boyden Chamber at $37^{\circ} \mathrm{C}$ with $\mathrm{C} 5 \mathrm{a}$ in the lower chamber $(B$, $C$ ). PMN were collected from the upper compartment $(B)$ and lower compartment $(C)$. The horizontal axis depicts fluorescence intensity using the same scale as in Figure 2. The vertical axis depicts the number of PMN. The 31D8 MAb binding, as measured by mean channel fluorescent intensity, is as follows: control, 155; upper, 141; lower, 182.

by these glycoproteins, and PMN deficient in these glycoproteins have marked abnormalities in adherence-related functions including spontaneous movement and chemotaxis. In contrast to 31D8 MAb binding, there are no distinct PMN subpopulations observed with Mo-1 MAb binding to PMN. There is a range of Mo-1 binding intensity, but instead of a biphasic curve as is observed with $31 \mathrm{D} 8 \mathrm{MAb}$, a single Gaussian distribution exists. In this study, adult PMN had increased binding of Mo-1 compared with neonatal PMN when both were tested on the same d under conditions of cell activation (139 \pm 26 versus $122 \pm 75, p$ $=0.014, n=5$ ). The results of Mo-1 MAb binding to PMN from the upper and lower compartments of the chemotactic chamber after $180 \mathrm{~min}$ are summarized in Figure 4. In these experiments, evaluation of adult and neonatal PMN responses were carried out on separate days; thus comparisons were made between different PMN responses within the adult or neonatal groups rather than between neonatal and adult PMN responses. There was significantly decreased Mo- 1 MAb binding to PMN obtained from the upper compared with the lower compartment in adults $(n=5): 143 \pm 13$ versus $164 \pm 9$, respectively, $p<0.01$ and in neonates $(\mathrm{n}=3)$ : $140 \pm 12$ versus $171 \pm 22, p<0.05$. A similar pattern was noted in Mo-1 MAb binding to PMN from the upper and lower compartments at 45 min for adults $(n=5): 134 \pm 10$ versus $170 \pm 12, p<0.01$, but not for neonates $(n=3)$ : $127 \pm$ 2 versus $144 \pm 13, p=0.34$. As with $31 \mathrm{D} 8 \mathrm{MAb}$, all control PMN were intermediate in binding between poorly motile and motile PMN.

\section{DISCUSSION}

PMN have long been considered to be a homogeneous population of mature cells, but it is now recognized that PMN are heterogeneous in regard to motility, phagocytosis, oxidative metabolism, protein synthesis, cell density and membrane receptors for IgGFc, IgAFc and MAb (1-10). At present, no single model can explain the origin of PMN heterogeneity and the relationship between the various PMN subpopulations which have been described. In the present study, nonmotile and motile PMN were isolated in an attempt to clarify their relationship to other PMN subpopulations and investigate their origin.

Wide variations in PMN motility have been described as early as the 1920's (1-2). Although fewer than 5\% of PMN were found to be nonmotile in these studies, visual assays were used, and only random PMN migration was assessed. More recently, Howard (3) used a visual assay to observe PMN in the presence and absence of the chemoattractant PMLP and noted that $17-21 \%$ of the PMN had no chemokinetic response to fMLP. Harvath and Leonard (4) studied PMN heterogeneity using a polycarbonate micropore filter chemotactic assay. More than $95 \%$ of the PMN were found to be nonmotile when no chemoattractant was used, 60-80\% were nonmotile in response to fMLP, and 50-70\% were nonmotile with C5a. These results were in close agreement with those of Keller (5), who used a nitrocellulose micropore filter assay. The results of the present study regarding the size of the adult nonmotile PMN subpopulation (approximately 60\%) are similar to those of previous studies using the micropore filter chemotactic assay but are different than those using visual assays. These differences can be explained at least in part by differences in the methods used to assess PMN motility. Unlike the visual assays, not all PMN can be accounted for using the micropore filter chemotactic assays. We were unable to recover up to 30 $40 \%$ of PMN in some of our experiments. Much of this loss was due to adherent PMN, and wash experiments indicated that there was similar recovery of adherent PMN from the upper and lower compartments of the Boyden chamber. The micropore filter chemotactic assay is preferred over the visual assay, however, for isolation and further characterization of the nonmotile and motile PMN subpopulations. In addition to the difference in the visual and micropore filter chemotactic assays, it is apparent that the type of chemoattractant used can influence the size of the nonmigrating subpopulation. A larger percentage of nonmotile PMN was found when we used fMLP compared with C5a. As previously noted, PMN that failed to migrate to C5a did not migrate when an additional chemoattractant (fMLP) was added (4).

Previous studies have demonstrated that PMN subpopulations exist, but little is known about their origin. Potential sources of PMN heterogeneity include $I$ ) varying environmental conditions that PMN may encounter in the circulation, 2) the possibility that PMN subpopulations may be derived from different progenitor cells, and 3) possible maturational differences among PMN. Our data suggest that poorly motile PMN are less mature than motile PMN. The nonmotile cells had a significantly greater number of bands than the motile cells. This is not sufficient to 
NEUTROPHIL SUBPOPULATIONS

Table 1. PMN $31 D 8$ antigen expression in chemotactic subpopulations*

\begin{tabular}{|c|c|c|c|c|c|c|c|c|}
\hline \multirow[b]{2}{*}{ Subject } & \multirow[b]{2}{*}{$\begin{array}{l}\text { Incubation } \\
\text { time (min) }\end{array}$} & \multirow[b]{2}{*}{$n$} & \multicolumn{2}{|c|}{ Upper compartment } & \multicolumn{2}{|c|}{ Lower compartment } & \multicolumn{2}{|c|}{ Control } \\
\hline & & & $\begin{array}{l}\text { Mean \% } \\
31 \text { D8 dull }\end{array}$ & $\begin{array}{c}\text { Mean } \\
\text { channel }\end{array}$ & $\begin{array}{l}\text { Mean } \% \\
\text { 31D8 dull }\end{array}$ & $\begin{array}{c}\text { Mean } \\
\text { channel }\end{array}$ & $\begin{array}{l}\text { Mean \% } \\
3108 \text { dull }\end{array}$ & $\begin{array}{c}\text { Mean } \\
\text { channel }\end{array}$ \\
\hline \multirow[t]{3}{*}{ Adult } & 45 & 5 & $7 \pm 1$ & $166 \pm 7 \dagger$ & $5 \pm 3$ & $184 \pm 14$ & $7 \pm 1$ & $165 \pm 10$ \\
\hline & 180 & 5 & $9 \pm 3$ & $157 \pm 9 \dagger$ & $8 \pm 5$ & $180 \pm 9$ & $9 \pm 3$ & $164 \pm 14$ \\
\hline & $45-180$ & 10 & $8 \pm 2$ & $166 \pm 7 \uparrow$ & $7 \pm 4$ & $183 \pm 12$ & $8 \pm 2$ & $164 \pm 11$ \\
\hline \multirow[t]{3}{*}{ Neonate } & 45 & 3 & $23 \pm 8$ & $152 \pm 3 \ddagger$ & $20 \pm 1$ & $173 \pm 15$ & $24 \pm 8$ & $157 \pm 8$ \\
\hline & 180 & 4 & $32 \pm 12 \S$ & $148 \pm 18 \ddagger$ & $15 \pm 5$ & $179 \pm 10$ & $28 \pm 14$ & $159 \pm 9$ \\
\hline & $45-180$ & 7 & $28 \pm 11 \ddagger$ & $150 \pm 12 \dagger$ & $17 \pm 5$ & $176 \pm 12$ & $26 \pm 11$ & $158 \pm 8$ \\
\hline
\end{tabular}

* All values shown represent mean $\pm 1 \mathrm{SD}$. The difference between mean values of the upper $v s$. lower compartment are indicated below.

$\dagger p<0.01$.

$\ddagger p<0.05$

$\S p=0.064$.

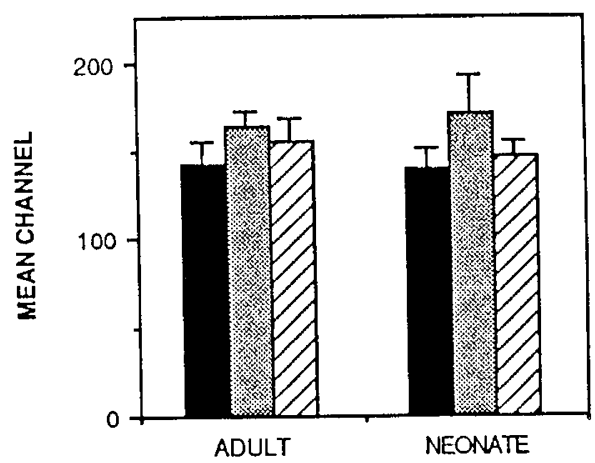

Fig. 4. PMN Mo-1 antigen expression in PMN chemotactic subpopulations. In separate experiments on different days, adult and neonatal PMN were allowed to traverse a polycarbonate micropore filter in a chemotactic chamber for 180 min. PMN were harvested from the upper and lower compartments of the chamber, labeled with Mo-1, and analyzed by flow cytometry. There was decreased Mo-1 expression in nonmigrating PMN (upper compartment, (lower compartment, 圈) as shown by a significantly decreased mean channel number in this subpopulation in neonates $(140 \pm 12$ vs. $171 \pm$ $22, p<0.05)$ and in adults $(143 \pm 13$ vs. $164 \pm 9, p<0.01)$. Mo- 1 antigen expression in control PMN (שas) was intermediate.

explain the difference between the two cell groups as there were not enough bands (or less mature forms such as myelocytes or metamyelocytes) to account for the large percentage of poorly motile cells; however, it is possible that the increased bands in the nonmotile cell group may be a marker for a less mature subpopulation of PMN. The decreased expression of 31D8 MAb in the poorly motile PMN subpopulation suggests that the nonmotile PMN are less mature than the motile PMN subpopulation. The 31D8 MAb was first produced by immunizing BALB/ $c$ mice with human PMN, fusing the spleen cells of these mice with a myeloma cell line and selecting for an anti-PMN antibody that bound PMN heterogeneously (9). The original characterization of the 31D8 antigen indicated that there was increased expression of the antigen during myeloid maturation from the promyelocyte to the mature PMN. Recent preliminary studies in our laboratory have confirmed and extended these findings (17). A study in patients suffering blunt trauma indicated that while PMN with decreased expression of 31D8 antigen (31D8 "dull") appear to be morphologically mature, they are, in fact, less mature than 31D8 "bright" PMN (18). Previous studies of the expression of the Mo-1, LFA-1, p1 50,95 glycoproteins during leukocyte differentiation in HL60 cells, U937 cells, and bone marrow suggest that, like $31 \mathrm{D} 8$ antigen, Mo-1 is increasingly expressed during myeloid development (19-20). Thus, if there is continued maturation of 31D8 and Mo-1 antigen expression in PMN from the bone marrow and peripheral blood, the less mature PMN subpopulation would be expected to express less
31D8 and Mo- 1 antigen than the more mature PMN subpopulation. Finally, although it is possible that motile and nonmotile PMN subpopulations are a result of varying environmental effects, their similarity in regard to NBT reaction and trypan blue exclusion indicate that environmental factors causing cell activation or death do not account for these subpopulations. Previous studies have shown that neither C5a, fMLP, phorbol myristate acetate, or opsonized zymosan cause changes in PMN 31 D8 antigen expression $(9,10,17,21)$. Thus, it is unlikely that different degrees of exposure to chemoattractant account for the differences noted between 31D8 antigen expression in the PMN from the top and bottom compartments of the Boyden chamber.

The results of this study suggest that the increased size of a poorly motile PMN subpopulation in neonates may help account for their decreased PMN motility and increased susceptibility to infection (22-26). Other studies have indicated that the increased size of a poorly functioning PMN subpopulation may help explain decreased PMN function in the human fetus, neonate, and in individuals suffering blunt trauma and burns $(10,18,27-$ 30). The cause for the increased nonmotile PMN subpopulation in neonates is unclear but may be due to the altered location and kinetics of PMN formation in neonates $(31,32)$. Further studies to clarify the origin of PMN subpopulations that may improve our understanding of defective PMN function in neonates and other compromised hosts are in progress.

Acknowledgments. We thank Carol Guiliano, Karen Davison, Kathy Kosciol, Jeff Novick, Rustin Levy, and David Brault for technical assistance, and Gail Frese for help with preparation of the manuscript.

\section{REFERENCES}

1. Gallin JI 1984 Human neutrophil heterogeneity exists, but is it meaningful? Blood 63:977-983

2. Sabin FB 1923 Studies of living human blood cells. Johns Hopkins Hospital Bull 34:277-281

3. Howard TH 1982 Quantification of the locomotive behaviour of polymorphonuclear leukocytes in clot preparations. Blood 59:946-951

4. Harvath L, Leonard EG 1982 Two neutrophil populations in human blood with different chemotactic activities: separation and chemoattractant bind. ing. Infect Immun 36:443-449

5. Keller HU, Wissler JH, Damerau 1981 Diverging effects of serum peptides and synthetic f-met-leu-phe on neutrophil locomotion and adhesion. Immunology 42:379-383

6. Ramsey WS 1972 Analysis of individual leukocyte behavior during chemotaxis Exp Cell Res 70:129-139

7. Klempner MS, Gallin JI 1978 Separation and functional characterization of human neutrophil subpopulations. Blood 51:659-669

8. Berkow RL, Baehner AL 1985 Volume-dependent human blood polymorphonuclear leukocyte heterogeneity demonstrated with counterflow centrifugal elutriation. Blood 65:71-78

9. Seligmann B, Malech HL, Melnick DA, Gallin JI 1985 An antibody binding to human neutrophils demonstrates antigenic heterogeneity detected early in myeloid maturation which correlates with functional heterogeneity of mature neutrophils. J Immunol 135:2647-2653

10. Krause PJ, Malech HL, Kristie J, Kosciol CM, Herson VC, Eisenfeld L, Pastuszak WT, Kraus A, Seligmann B 1986 Polymorphonuclear leukocyte 
heterogeneity in neonates and adults. Blood 68:200-204

11. Anderson EC, Springer TA 1987 Leukocyte adhesion deficiency: an inherited defect in the MAC-1, LFA-1, and p150,95 glycoproteins. Annu Rev Med 38:175-194

12. Bruce MC, Baley JE, Medvik KA, Berger M 1987 Impaired surface membrane expression of $\mathrm{C} 3 \mathrm{bi}$ but not $\mathrm{C} 3 \mathrm{~b}$ receptors on neonatal neutrophils. Pediatr Res 21:306-31

13. Anderson DC, Freeman KL, Heerdt B, Hughes BJ, Jack RM, Smith CW 1987 Abnormal stimulated adherence of neonatal granulocytes: impaired induction of surface MAC-1 by chemotactic factors or secretagogues. Blood 70:740-750

14. Krause PJ, Pock RM, Woronick CL, Maderazo EG 1983 Simplified micropore filter assay of neutrophil migration using whole blood. J Infect Dis 148:881 885

15. Metcalf JA, Gallin JI, Nauseef WM, Root RK 1986 Laboratory Manual of Neutrophil Function. Raven Press, New York

16. Krause PJ, Maderazo EG, Scroggs M 1982 Abnormalities of neutrophil adherence in newborns. Pediatrics 69:184-187

17. Krause PJ, Todd M, Pastuszak W, Malech HM 1988 A surface antigen associated with cell function is increasingly expressed during myeloid development. Program and abstracts of the 28th Interscience Conference on Antimicrobial Agents and Chemotherapy, Los Angeles, CA 18:280(abstr)

18. Krause PJ, Maderazo EG, Bannon P, Kosciol K, Malech HM 1988 Neutrophil heterogeneity in patients with blunt trauma. J Lab Clin Med 112:208-215

19. Miller BA, Antognetti G, Springer TA 1985 Identification of cell surface antigens present on murine hematopoietic stem cells. J Immunol 134:32863290

20. Miller LJ, Schwarting R, Springer TA 1986 Regulated expression of the Mac1, LFA-1, p150,95 glycoprotein family during leukocyte differentiation. J Immunol 137:2891-2900
21. Hickstein DD, Locksley RM, Beatty PG, Smith A, Stone DM, Root RK 1986 Monoclonal antibodies binding to the human neutrophil C3bi receptor have disparate functional effects. Blood 67:1054-1062

22. Miller ME, Stiehm ER 1983 Immunology and resistance to infection. In Remington JS, Klein JD (eds) Infectious Diseases of the Fetus and Newborn Infant. WB Saunders, Philadelphia, pp 27-68

23. Hill HR 1987 Biochemical, structural, and functional abnormalities of poly morphonuclear leukocytes in the neonate. Pediatr Res 22.375-382

24. Miller ME 1971 Chemotactic function in the human neonate: humoral and cellular aspects. Pediatr Res 5:487-492

25. Anderson DC, Hughes BJ, Smith CW 1971 Abnormal mobility of neonata polymorphonuclear leukocytes. J Clin Invest 68:683-874

26. Krause PJ, Herson VC, Boutin-Lebowitz J, Eisenfeld L, Block C, LoBello T, Maderazo EG 1986 Polymorphonuclear leukocyte adherence and chemotaxis in stressed and healthy neonates. Pediatr Res 20:296-300

27. Smith JB, Campbell DE, Douglas SD, Garty BZ, Ludomirsky A, Polin RA, Harris MC 1988 Fetal neutrophils have distinct subpopulations which differ in complement and Fc receptor expression. Pediatr Res 23(Suppl):468(abstr)

in complement and Fc receptor expression, Pedatr Res 23(Suppl):468(abstr) thermal injury. J Trauma 26:534-537

29. Hilmo A Howard TH 1987 F-Actin content of neonate and adult neutrophils. Blood 69:945-949

30. Masuda K, Kinoshita Y, Kobayashi Y 1988 Heterogeneity of Fc receptor expression in chenotaxis and adherence of neonatal neutrophils. Pediatr Res 25:6-10

31. Christensen RD, Rothstein G 1984 Pre- and postnatal development of granulocyte stem cells in the rat. Pediatr Res 18:599-602

32. Christensen RD, Rothstein G 1985 Neutrophil myeloperoxidase concentration: changes with development and during bacterial infection. Pediatr Res 19:1278-1282 